\title{
Towards the final fate of an unstable black string
}

\author{
Matthew Choptuik, ${ }^{1,2}$ Luis Lehner, ${ }^{3,2}$ Ignacio (Tnaki) Olabarrieta, ${ }^{2}$ Roman Petryk, ${ }^{2}$ Frans Pretorius, ${ }^{4,2}$ and Hugo Villegas ${ }^{2}$ \\ ${ }^{1}$ CIAR Cosmology and Gravity Program, Canada \\ ${ }^{2}$ Department of Physics and Astronomy, University of British Columbia, Vancouver, Canada V6T 1Z1 \\ ${ }^{3}$ Department of Physics and Astronomy, Louisiana State University, Baton Rouge, Louisiana 70810, USA \\ ${ }^{4}$ Theoretical Astrophysics 130-033, California Institute of Technology, Pasadena, California 91125, USA
}

(Received 28 April 2003; published 1 August 2003)

\begin{abstract}
Black strings, one class of higher dimensional analogues of black holes, were shown to be unstable to long wavelength perturbations by Gregory and Laflamme in 1992, via a linear analysis. We reexamine the problem through the numerical solution of the full equations of motion, and focus on trying to determine the end state of a perturbed, unstable black string. Our preliminary results show that such a spacetime tends towards a solution resembling a sequence of spherical black holes connected by thin black strings, at least at intermediate times. However, our code fails then, primarily due to large gradients that develop in metric functions, as the coordinate system we use is not well adapted to the nature of the unfolding solution. We are thus unable to determine how close the solution we see is to the final end state, though we do observe rich dynamical behavior of the system in the intermediate stages.
\end{abstract}

DOI: 10.1103/PhysRevD.68.044001

PACS number(s): 04.50.+h, 04.25.Dm, 04.70.Bw

\section{INTRODUCTION}

The stability of four-dimensional black holes is a well known and fundamental result of relativity theory [1]. The picture in higher dimensional spacetimes was shown to be quite different by Gregory and Laflamme [2,3], who demonstrated the existence of unstable long wavelength modes of the black string in perturbation theory. This finding, coupled to arguments based on entropy considerations, led to their conjecture that black strings might bifurcate into higher dimensional analogues of spherical black holes. Cosmic censorship would be violated were this the case, since a singularity must be encountered by a bifurcating black hole horizon, essentially as a consequence of the principle of equivalence [4].

The existence of the Gregory-Laflamme instability has been assumed in many subsequent studies of higher dimensional gravity theory, including the classical limit of string theory (see, for example, [5-8]), some of which have also assumed the validity of the bifurcation conjecture. However, a linearized analysis can say little, if anything, concerning the nature of the full non-linear evolution of an unstable string, and the final end state of such a configuration remained to be established.

Recently, Horowitz and Maeda were able to prove, under some assumptions, that black strings cannot bifurcate in finite time [9]. Furthermore, they conjectured that the system is likely to approach a new stationary solution which is not translationally invariant along the string direction. However, even if the assumptions involved in the proof are sufficiently generic, their analysis cannot identify the final end-state of evolution. Partial answers can be sought via perturbation analysis as done by Gubser [10]. By assuming the HorowitzMaeda conjecture and linearizing the solution at the critical length to first order (and a partial extension to second order), Gubser argued that the transition to the final solution must be of second order type (i.e. discontinuous). Despite these developments, it seems clear that a convincing answer to the question at hand can only be obtained by solving the full equations governing the problem. A step in this direction would be to search for special solutions, such as stationary ones, and compare the physical content of the obtained configurations with the black string solutions. This has recently been carried out by Wiseman [11], who numerically solves the equations resulting from a static ansatz. Interestingly, he finds non-uniform solutions with mass larger than that of the black string for a given compactification radius. Thus, he concludes that the solutions he finds cannot be the end states conjectured by Horowitz and Maeda (also see related work by Kol [12]).

Additional work by Unruh and Wald [13] studies the dynamics of a uniform cylindrical configuration of matter in Newtonian gravity. They observe that a perturbation of the density gives raise to a Jeans instability responsible for the collapse of the system along the cylinder's length. They then argue that if the main features of this model are robust in the passage to the general relativistic system, one possible end state for the perturbed black string would be collapse in the string direction, resulting in singularity formation. Note that this collapse need not lead to violations of cosmic censorship, as the final singularity could still be hidden by an event horizon [14].

Clearly there are several distinct viable possibilities for the final end-state of a perturbed black string, with remarkably different consequences associated with the range of options. Current conjectures range from "nothing interesting happens," to violations of cosmic censorship, to the arguably more extreme case of a complete collapse of the spacetime. In order to completely settle the issue, the full dynamics of the perturbed black string needs to be addressed. At least in principle this will allow us to identify which of the above possibilities (if any) is actually realized. In this paper we report on preliminary work in this direction-a program to simulate the dynamics of the black string through numerical solution of the Einstein equations. At this stage of the project, we cannot yet provide an answer to the question of 
the end state; however, we have tantalizing results that show the spacetime going through a configuration resembling 5-dimensional spherical black holes connected by thin black strings that expand along the string dimension. Our simulations eventually crash while the spacetime is still fairly dynamical, and so we cannot determine whether what we see is near the end state, or merely an intermediate configuration in a more complicated evolution. Underlying the current failure of our simulations is the fact that the coordinate system employed is not well adapted to the solution that unfolds at late times, wherein fatally steep gradients develop in metric functions. ${ }^{1}$

The outline of the remainder of the paper is as follows. In Sec. II we begin by describing the equations of motion, our coordinate choices, the generation of initial data, as well as our numerical solution scheme. Additionally, we also briefly mention the tools we employ to monitor the solution, deferring details to the Appendixes. In Sec. III we discuss the results obtained with this code, and conclude in Sec. IV by mentioning directions for future work that may allow us to more definitively answer questions regarding the end state of the Gregory-Laflamme instability.

\section{EQUATIONS, BLACK STRINGS AND NUMERICS}

We wish to solve the vacuum Einstein equations in higher dimensional settings. For simplicity, and without loss of generality in studying the Gregory-Laflamme instability, we only consider the 5-dimensional case, and restrict attention to spherical symmetry within the 4-dimensional subspace tangent to the "extra" dimension. We also use the natural generalization of the Arnowitt-Deser-Misner (ADM) decomposition to derive the system of equations that we then solve numerically. Choosing units in which $G=c=1$, and adopting Misner-Thorne-Wheeler (MTW) [15] conventions, our starting point is thus a metric element given by

$$
\begin{aligned}
d s^{2}= & \left(-\alpha^{2}+\gamma_{A B} \beta^{A} \beta^{B}\right) d t^{2}+2 \gamma_{A B} \beta^{A} d x^{B} d t \\
& +\gamma_{A B} d x^{A} d x^{B}+\gamma_{\Omega} d \Omega^{2}
\end{aligned}
$$

where $x^{A}=(r, z)$, and $d \Omega^{2}$ is the 2-spherical line element with coordinates chosen orthogonal to the $t=$ const congruences (hence there is no shift corresponding to angular directions). All metric components defined via Eq. (1) depend upon $(t, r, z): t$ is a time-like coordinate, $r$ is a radial coordinate, and $z$ is the coordinate along the length of the string. To further expedite the numerical implementation, we make $z$ a periodic coordinate by identifying $z=0$ and $z=L$. Then, following the results of Gregory and Laflamme, we expect black strings to be unstable only for $L$ greater than some critical length $L_{c}$.

\footnotetext{
${ }^{1}$ This divergence of metric gradients does not happen earlier as resolution is increased, and is not accompanied by divergence of curvature invariants such as the Kretschmann scalar. This suggests that the code is evolving to a coordinate, rather than a geometric, singularity.
}

The vacuum Einstein equations, written in $\mathrm{ADM}$ form $[15,16]$, are (1) the Hamiltonian constraint

$$
H \equiv{ }^{(4)} R+K^{2}-K_{a b} K^{a b}=0,
$$

(2) the momentum constraints

$$
M_{a} \equiv K_{a \mid b}^{b}-K_{\mid a}=0
$$

(3) the evolution equations for the $\gamma_{a b}$

$$
\frac{\partial \gamma_{a b}}{\partial t}=-2 \alpha K_{a b}+\beta_{a \mid b}+\beta_{b \mid a},
$$

that follow from the definition of the extrinsic curvature $K_{a b}$ associated with $t=$ const slices, and (4) the evolution equations for the extrinsic curvature

$$
\begin{aligned}
\frac{\partial K_{a b}}{\partial t}= & \alpha\left({ }^{(4)} R_{a b}+K K_{a b}\right)-2 \alpha K_{a c} K^{c}{ }_{b}-\alpha_{\mid a b}+\beta^{c}{ }_{\mid a} K_{c b} \\
& +\beta^{c}{ }_{\mid b} K_{c a}+\beta^{c} K_{a b \mid c}+\alpha F_{a}^{c} F_{b}^{d} \gamma_{c d} H .
\end{aligned}
$$

In the above, $a, b, \ldots$ are four-dimensional (spatial) indices, ${ }^{(4)} R_{a b}$ and ${ }^{(4)} R$ are, respectively, the Ricci tensor and Ricci scalar intrinsic to the four-dimensional spatial hypersurfaces, $\alpha$ is the lapse function, $\beta^{c}$ is the shift vector, the vertical bar denotes covariant differentiation in the spatial hypersurfaces (compatible with $\gamma_{a b}$ ), and $F_{a}^{c}=-2 \delta_{r}^{c} \delta_{a}^{r}$. We note that the term proportional to $H$ in Eq. (5) has been added as a result of stability considerations; see, for instance, the discussions in [17-19]. To simplify the final set of equations solved numerically, as well as to regularize certain terms that otherwise diverge at spatial infinity (see the discussion of our coordinate system in the next section), we define the following variables:

$$
\begin{gathered}
g_{r r} \equiv \gamma_{r r}, \quad g_{r z} \equiv \gamma_{r z}, \quad g_{z z} \equiv \gamma_{z z}, \\
g_{\theta \theta} \equiv \gamma_{\Omega} / r^{2}, \quad g_{\phi \phi} \equiv \gamma_{\Omega} /\left(r^{2} \sin ^{2} \theta\right), \\
k_{r r} \equiv r^{2} K_{r r} / \alpha, \quad k_{r z} \equiv K_{r z}, \quad k_{z z} \equiv K_{z z}, \\
k_{\theta \theta} \equiv K_{\theta \theta} / \alpha, \quad k_{\phi \phi} \equiv K_{\theta \theta} /\left(\alpha \sin ^{2} \theta\right),
\end{gathered}
$$

and use them as the fundamental dynamical quantities in our numerical code. As discussed in the following sections, to complete the prescription of the evolution problem we need to choose a suitable lapse and shift, specify initial and boundary conditions, and then implement these choices and specifications consistently.

\section{A. Boundary and coordinate conditions}

A particular concern here is that "standard" outer boundary conditions [17], often imposed during numerical evolution of Einstein's equations, might not be well suited for studying the string instability. In particular, we must be able to evolve for very long times while absolutely minimizing spurious influences from the outer boundary of the computational domain. In addition, in the present case we cannot 
assume, a priori, that any given initial configuration will settle down to some stationary solution; thus, boundary conditions predicated on such assumptions (such as a $1 / r$ fall-off condition in a metric component), when imposed at a finite proper distance from the black string, could very well adversely affect the numerical results. ${ }^{2}$ To ensure minimal boundary influence we therefore extend the domain of integration to $i^{o}$ by radially compactifying the spacelike hypersurfaces via the introduction of a new coordinate, $x$, defined by

$$
x \equiv \frac{r}{1+r}
$$

As might be expected, this transformation causes computational problems of its own-most notably decreased spatial resolution at large distances-but, as discussed in Sec. II C, we can deal with these difficulties using numerical dissipation. Having introduced the new compactifying coordinate, we can directly impose boundary conditions derived from the demand of asymptotic flatness at spatial infinity, which lies at $x=1$.

We employ singularity excision techniques [20] to allow us to evolve the entire perturbed black string spacetime exterior to the apparent horizon (plus a certain "buffer zone" that lies within the horizon). Hence, we do not need to impose inner boundary conditions as long as the $t=$ const hypersurfaces penetrate the horizon, and that all characteristics of the evolution equations are in-going on the boundary. Ensuring that this is the case involves choosing "good" coordinate conditions (choice of lapse and shift), which, for generic string evolutions, remains an open problem. As a preliminary step, we have based our coordinate choices on those that yield the ingoing Eddington-Finkelstein form of the unperturbed black string metric

$$
\begin{aligned}
d s_{\mathrm{BS}}^{2}= & -(1-2 M / r) d t^{2}+4 M / r d r d t+(1+2 M / r) d r^{2} \\
& +d z^{2}+r^{2} d \Omega^{2}
\end{aligned}
$$

Comparison with the general 5-dimensional ADM form provides the identifications

$$
\begin{aligned}
& \alpha_{\mathrm{BS}}=(1+2 M / r)^{(-1 / 2)}, \\
& \beta_{\mathrm{BS}}^{A}=(2 M /(r+2 M)) \delta_{r}^{A} .
\end{aligned}
$$

For reference we also list the two non-trivial components of the extrinsic curvature of a $t=$ const slice defined by Eq. (8):

$$
K_{r r}=-2 M \frac{(r+M)}{r^{3}} \sqrt{\frac{r}{r+2 M}}
$$

\footnotetext{
${ }^{2}$ In fact, in an earlier version of the code that did not use a radially compactified coordinate system, we did encounter such problems, in that some artificial stationary non-homogeneous solution was apparently entirely "sourced" via an outer boundary located at a finite distance from the string.
}

$$
K_{\theta \theta}=2 M \sqrt{\frac{r}{r+2 M}}
$$

In generalizing Eqs. (9) and (10) to the dynamical case, we have chosen $\alpha=\alpha_{\mathrm{BS}}$ and $\beta^{z}=\beta_{\mathrm{BS}}^{z}=0$. In a preliminary version of our code, we also required that $\beta^{r}=\beta_{\mathrm{BS}}^{r}$. This, however, caused a coordinate pathology to develop at late times during the evolution of unstable strings-specifically, some regions of the horizons approached a zero coordinate radius, while maintaining finite proper radius. In our current efforts, we choose $\beta^{r}$ such that $g_{\theta \theta}$ remains constant during evolution $[21,22]$, by requiring that

$$
\beta^{r}=\frac{2 \alpha K_{\theta \theta}}{\gamma_{\theta \theta, r}} .
$$

This shift condition performs reasonably well, as will be seen in Sec. III. However, our current simulations still suffer from "grid-stretching" problems in the $z$ direction at late times, suggesting that a more dynamical gauge condition for $\beta^{z}$ (and possibly for $\alpha$ and $\beta^{r}$ ) could be useful. This issue is discussed in more detail in Sec. IV.

\section{B. Initial data}

As anticipated, we observe that even numerical truncation errors, if non-uniform in the $z$ direction, are enough to trigger the Gregory-Laflamme instability in our simulations. However, to reduce the computational effort required to reach the "interesting" (i.e. non-perturbative) stages of evolution, we adopt initial configurations whose departure from the black string solution can be arbitrarily tuned. In order to find such data, we must solve the Hamiltonian constraint, and the $r$ and $z$ components of the momentum constraint (the other components of the momentum constraint are trivially satisfied because our coordinate system is adapted to spherical symmetry). The deviation-not necessarily small—from the black string solution, is introduced via $g_{\theta \theta}$, and takes the following form:

$$
g_{\theta \theta}(0, r, z)=1+A \sin \left(z \frac{2 \pi q}{L}\right) e^{-\left(r-r_{o}\right)^{2} / \delta_{r}^{2}} .
$$

Here, $A$ is used to control the overall strength of the "perturbation," while $q$ is an integer that controls the spatial frequency in the $z$ direction. For the results presented below, $A=0.1, q=1, r_{0}=2.5$ and $\delta_{r}=0.5$, and we perturb about a unit mass $(M=1)$ black string solution. As described in more detail in Appendix A, $g_{r r}, k_{r r}, k_{\theta \theta}$ are then calculated by solving the constraint equations, with the remainder of the metric and extrinsic curvature variables set to the values they would take for an unperturbed black string [see Eqs. (8) and (11)].

\section{Numerical evolution}

To numerically evolve the initial data sets described above, we discretize the evolution equations (4), (5) using second-order accurate finite difference techniques that include the Crank-Nicholson treatment of the temporal and 
spatial derivatives. We use a uniform distribution of grid points in $z$ and $x$ [recall that $x \equiv r /(1+r)$ ]. The resulting implicit system of algebraic equations is solved iteratively. We initially implemented a serial version of the algorithm, and later coded a parallel version using the CACTUS Computational Toolkit [23], wherein the equations of motion, monitoring tools and $\mathrm{I} / \mathrm{O}$ were handled by our own routines, suitably interfaced to CACTUS.

Black hole excision is handled as follows. We periodically find the apparent horizon, as discussed in the following section and Appendix B. We then define the surface along which we excise to be a certain number of "buffer" points inside the apparent horizon (typically 10-30 buffer points are used). ${ }^{3}$ During each Crank-Nicholson iteration, all the evolution difference equations are applied up to the excision surface, and any function values referenced by finite difference stencils interior to this surface are defined via fourth order extrapolation. When the apparent horizon location and hence excision surface changes during evolution, function values at all repopulated points (i.e. those that moved from inside to outside the excision surface during the time step) are computed via the same fourth order extrapolation routine. The one exception to this procedure is for the grid values of $g_{\theta \theta}$, which we specify a priori on the entire computational domain, and that remain fixed due to our gauge choice (12). Moreover, we have found it useful to choose a functional form for $g_{\theta \theta}$ that tends to zero at some positive value of $r$ (though inside the original apparent horizon location and outside of the limits of integration of the initial data). This causes the "pinching off" of the unstable black string to be less severe in coordinate space, i.e. we approach zero areal radius at a finite coordinate $r$. In turn, this slightly reduces the virulence of the coordinate problems we observe at late times, and also provides better load balancing of the parallel code, given the method CACTUS uses to distribute grids among processors.

For the evolution, we choose a time step $d t$ $=\lambda_{\mathrm{CFL}} \min (d r, d z)$, where the constant $\lambda_{\mathrm{CFL}}$ must be set less than $1 / \sqrt{2}$ in order to satisfy the Courant-Friedrichs-Lewy (CFL) stability condition that results from our iterative solution of the Crank-Nicholson scheme (typically we use $\lambda_{\mathrm{CFL}}$ $=0.25$ ). Note that this restriction on $\lambda_{\mathrm{CFL}}$ is based upon flat-space light speeds within our coordinate system [Eq. (8) with $M=0$ ], which, for the solutions presented here, are always greater than or equal to the actual coordinate light speeds. The function $\min (d r, d z)$ is calculated by only considering mesh spacings within the non-excised portion of the coordinate domain. Thus, as the excision surface moves, $d t$ changes with time since our grid is uniform in $x$, and hence non-uniform in $r$.

Crucially, we add Kreiss-Oliger-style [24] numerical dissipation to the evolution equations to control unphysical

\footnotetext{
${ }^{3}$ In several tests, we also adopted an excision region given by the global minimum $r$ value of the apparent horizon and compared the results with those obtained when the excision region was defined by the apparent horizon. The agreement obtained gives extra indication that the excision implementation is consistent.
}

high-frequency solution components ("noise") that would otherwise arise during the simulations. This is particularly helpful at the excision surface, and near $i^{0}$, where the radial compactification of points causes all outgoing wave-like components of variables to eventually become poorly resolved. Smoothing of the high frequency components via the Kreiss-Oliger dissipation - which only targets wavelengths of size on the order of the mesh spacing-prevents them from inducing numerical instabilities near the outer boundary.

\section{Monitoring the evolution}

To elucidate the nature of our computed spacetimes, we monitor the following quantities: (1) the location of the apparent horizon (which is also used for excision as explained earlier), (2) the trajectories of null geodesics that, to a certain extent, should trace the event horizon, and (3) the Kretschmann invariant $I$ (the square of the Riemann tensor):

$$
I=R_{\alpha \beta \gamma \delta} R^{\alpha \beta \gamma \delta}
$$

If cosmic censorship holds-and results from our current simulations provide no evidence to the contrary-then any apparent horizon found will always be inside an event horizon. As is well known, although the apparent horizon can often be used as a reasonable approximation to the event horizon, the two do not, in general, coincide. ${ }^{4}$ Clearly, the event horizon is the quantity of interest in studying the Gregory-Laflamme instability, and therefore we would like to locate it, or at least a reasonably good approximation to it, in our simulation results. Such an approximation can be obtained by looking for the boundary of the causal past of some $r=$ const surface that is sufficiently far outside the apparent horizon that it is certain not to be inside the event horizon, yet close enough to the apparent horizon that its causal past, tracing backwards from the end time of the simulation, probes the region of interest of the spacetime. We use a method to find the approximate event horizon discussed by Libson et al. [25]. The approach is based on radial outgoing null geodesics; as explained in [25], the stable direction for the integration of null rays that emanate from the vicinity of an event horizon is backwards in time $t$. Thus, once we have the complete data from the entire evolution, we start with data from the latest time step available, and trace the null rays backwards in time.

Monitoring curvature scalars is useful in obtaining coordinate independent information about a numerical solution of the Einstein equations. In particular, $I$, as defined by Eq. (14), evaluates to $I_{\mathrm{BS}}=48 \mathrm{M}^{2} / \gamma_{\theta \theta}{ }^{3}$ for the unperturbed black string solution, and as $\gamma_{\theta \theta}$ is an invariant in spherical symmetry (i.e. it is proportional to the area of an $r=$ const 2 -sphere), we can compare $I_{\mathrm{BS}}$ to the values of $I$ computed from a numerical solution to get some indication of how close the computed solution is to a black string spacetime. Furthermore, we can examine $I$ to see whether curvature sin-

\footnotetext{
${ }^{4}$ Indeed, depending on the slicing an apparent horizon need not exist at all [26].
} 
gularities (other than the central $r=0$ singularity) may be forming prior to the demise of the simulation that invariably occurs when sufficiently steep metric gradients develop. We note, however, that if I does not diverge, it does not necessarily follow that the geometry is remaining non-singular; we would need to examine a larger set of curvature scalars to be certain that the solutions are remaining free of physical singularities.

Appendix B contains an explanation of the method we used to find apparent horizons, while details of the integration techniques aimed at approximately locating event horizons can be found in Appendix C.

\section{RESULTS}

In this section we present results from our preliminary study of the black string instability. After briefly showing that we recover some of the key Gregory-Laflamme results in the next section, we present a detailed analysis of a typical unstable case in Sec. III B. In the following, we will use the value of $L_{c} \approx 14.3 M$ (with $M=1$ ) found by Gubser [10], which is more accurate than the value we can estimate from the zero crossing of the (positive mode) interpolating curve presented in [2].

\section{A. Recovery of Gregory-Laflamme results}

We ran a variety of simulations of black strings that were perturbed according to the prescription discussed in Sec. II B. We concentrated on cases with $L$ ranging from $0.6 L_{c}$ to $1.8 L_{c}$ and defined $g_{\theta \theta}$ via Eq. (13). In general, we observed the expected instability for $L>L_{c}$, though for the maximum resolution at which we performed this survey (800 grid points in $r$ and 200 points in $z$ ), we could only confirm $L_{c}$ to within about $2 \%$ of the expected value. In this regard we note that as $L$ approaches $L_{c}$ from above, the growth rate of the instability goes to 0 , requiring longer time integrations to identify the instability, which, in turn, demands an ever increasing resolution to counter the effects of accumulating numerical errors. Furthermore, the initial configurations we have adopted contain energy in the form of gravitational waves, and some of this energy falls into the string early on during the evolution. The increase in the mass of the string (based upon the increase in area of the apparent horizon) is typically around $0.3-0.5 \%$, and we would have to take this into account were we to attempt to determine $L_{c}$ from our simulations to a higher degree of accuracy. Note, however, that for the purpose of this study, the only physically significant aspect of the initial gravitational radiation is to provide a mechanism to perturb an otherwise static black string.

As a demonstration of the ability of our code to "bracket" the instability, and following the notation of [10], Fig. 1 shows a plot of $\lambda$, defined by

$$
\lambda=\frac{1}{2}\left(\frac{R_{\max }}{R_{\min }}-1\right)
$$

for $L=0.975 L_{c}$ and $L=1.03 L_{c}$. In the above, $R_{\max }$ and $R_{\min }$ are the maximum and minimum areal radii, respectively, of

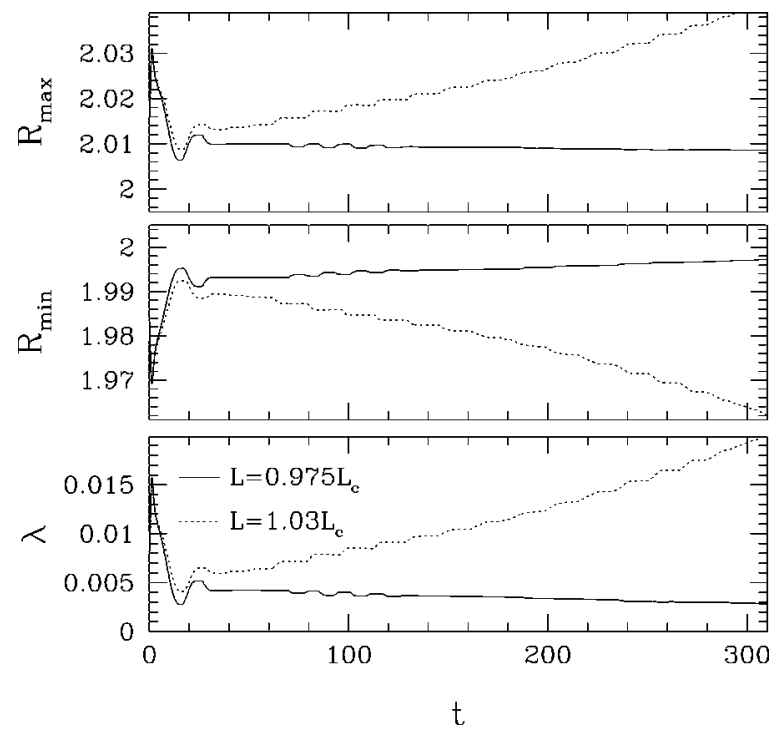

FIG. 1. The maximum $\left(R_{\max }\right)$ and minimum $\left(R_{\min }\right)$ areal radii, and the corresponding function $\lambda$ of the apparent horizon as a function of time, from the evolution of perturbed black strings with $L$ $=1.03 L_{c}$ and $L=0.975 L_{c}$. The initial fluctuation in the plots correspond to the effect of the initial gravitational wave perturbation, most of which either falls into the string or escapes to infinity. This close to the threshold $L_{c}$, the growth/decay of the remnant perturbation is quite slow, and so we cannot feasibly (at the resolution of the these simulations $-800 \times 200$ points in $r \times z$ ) follow the evolution for much further than shown while maintaining reasonable accuracy (though we see no signs of numerical instabilities in the stable case, and such simulations have been followed to $10000 M$ ). However, the main purpose of this figure is to demonstrate the qualitative recovery of the expected threshold behavior for the onset of the instability at $L=L_{c}$.

the apparent horizon at some $t=$ const slice of the spacetime. In particular, we have $\lambda=0$ for the static black string spacetime.

\section{B. Beyond the linear regime}

We now present more detailed results from the simulation of an unstable black string evolution. Specifically, we take $L=1.4 L_{c}$, since it is expected that this particular range for the $z$ coordinate will yield something close to the fastest growth rate for the shortest wavelength instability [2]. Because we are now probing uncharted territory with our computations, we rely on convergence tests to provide an intrinsic measure of the level of error in our calculations. To that end, we ran the simulation at several resolutions $\left(n_{r} \times n_{z}\right)$ : $200 \times 50$ (grid spacing $h), 400 \times 100(h / 2), 800 \times 200(h / 4)$, and $1600 \times 400(h / 8)$. Due to our use of a compactified radial coordinate, the lowest resolution calculation cannot adequately resolve the late-time behavior of the solution. However, for the "medium resolution" computation with mesh spacing $h / 4$, we are apparently within the convergent regime-see Fig. 2 below for plots of the maximum and minimum areal radii of the apparent horizon as a function of time, as well as the quantity $\lambda$ defined by Eq. (15), and Fig. 3 for plots of the norm of the Hamiltonian constraint as a 


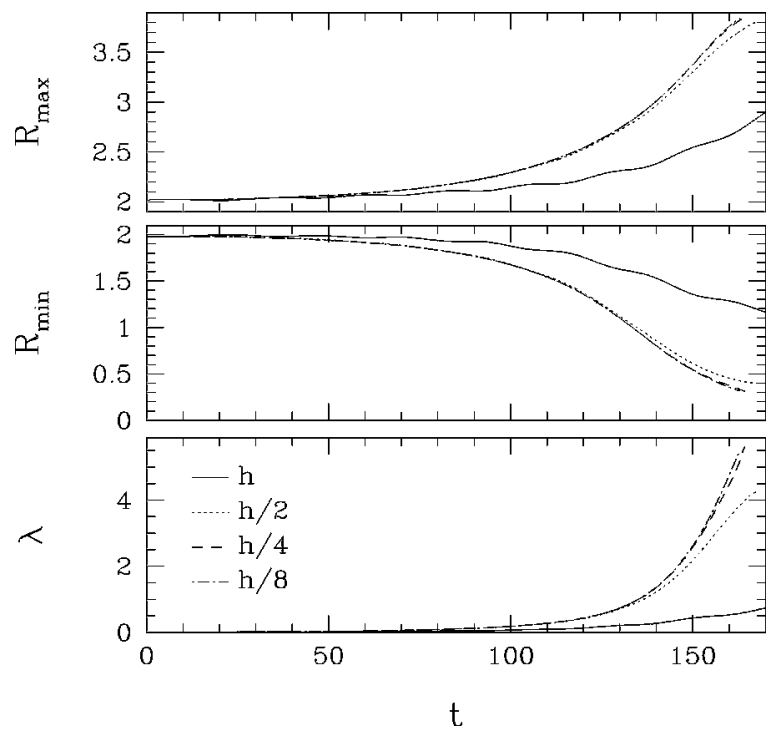

FIG. 2. The maximum $\left(R_{\max }\right)$ and minimum $\left(R_{\min }\right)$ areal radii, and the corresponding function $\lambda=\left(R_{\max } / R_{\min }-1\right) / 2$ of the apparent horizon, as a function of time, from the evolution of a perturbed black string with $L=1.4 L_{c} . h$ labels grid spacing; hence smaller $h$ corresponds to higher resolution. This plot, combined with the results shown in Fig. 3, suggest that the code is in the convergent regime-in particular at later times-for the $h / 2$ and higher resolution simulations.

function of resolution. Therefore, unless otherwise noted, all the results shown below are taken from the $h / 4$ simulation.

Figure 4 shows embedding diagrams of the apparent horizon at several times during evolution of the string, and Fig.

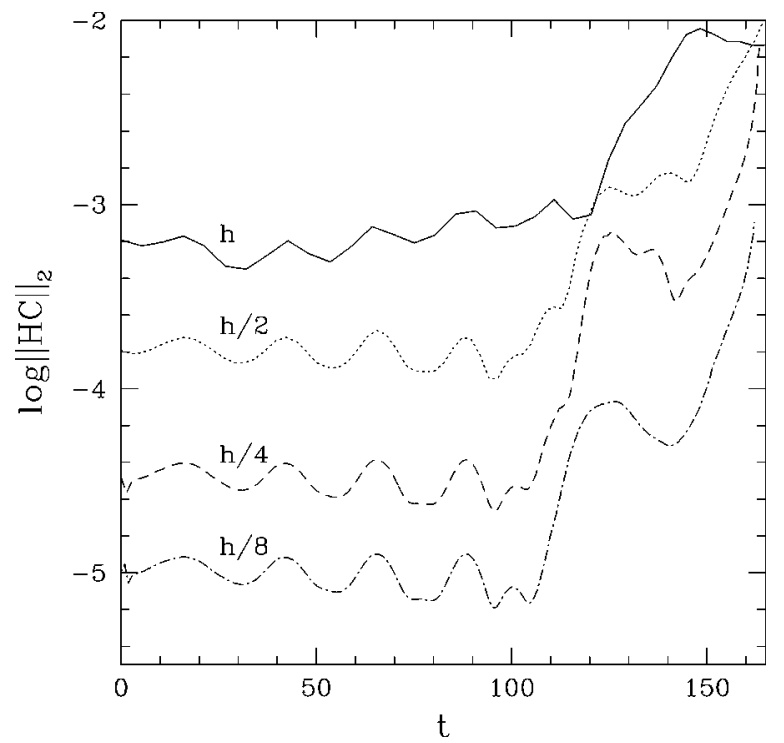

FIG. 3. The logarithm of the $\ell_{2}$-norm of the Hamiltonian constraint as a function of time, evaluated on the portion of the computational domain lying exterior to the apparent horizon, and from simulations at several resolutions of a perturbed black string with $L=1.4 L_{c}$. As with Fig. 2 , this plot provides evidence that convergence is quite good for the $h / 2$ and higher resolution simulations (at least until very close to when the supposed coordinate singularity forms, near $t=165$ ).

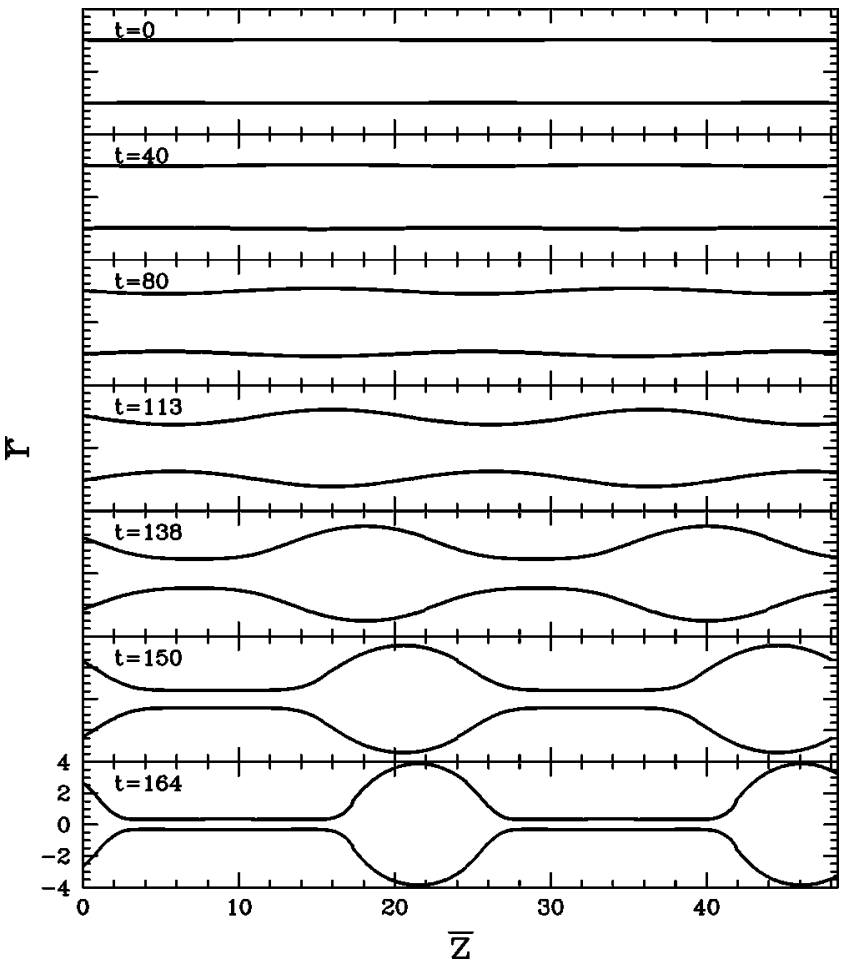

FIG. 4. Embedding diagrams of the apparent horizon, with the two angular dimensions $\theta$ and $\phi$ suppressed, from the $h / 4$ evolution of a perturbed black string with $L=1.4 L_{c}$. These plots thus describe the intrinsic geometry of the apparent horizon, at the given instants of constant $t$, in a coordinate system with metric $d s^{2}$ $=d \bar{r}^{2}+d \bar{z}^{2}$. Here, $\bar{z}$ is a periodic coordinate, and $\bar{r}$ is the areal radius of $\bar{z}=$ const sections of the horizon. To better illustrate the dynamics of the horizon, we have extended the solution using the $\bar{z}$ periodicity, showing roughly two periods of the solution. See Fig. 5 for a plot of the length of one period of the apparent horizon versus time.

5 shows the proper length of one period of the apparent horizon (suppressing the angular coordinates) versus time. (Our embedding uses the vertical axis to represent the areal radius of the apparent horizon - the horizontal axis is then uniquely determined by requiring that the length of the curve be equal to the proper length of the horizon.) The simulation crashes shortly after the last time frame shown, apparently due to the coordinate pathologies that have been discussed previously. The embedding diagrams suggest that, at least in the vicinity of the apparent horizon, the solution is tending towards a spacetime that can be described as a sequence of spherical black holes connected by thin black strings. Additional, quantitative, evidence for this conjecture can be obtained through a computation of the curvature invariant, $I$, on the apparent horizon. For an exact black string solution, this quantity, which we denote $I_{\mathrm{BS}}^{0}$, is

$$
I_{\mathrm{BS}}^{0}=\frac{12}{R_{\mathrm{AH}}^{4}}
$$

while for the 5-dimensional spherical black hole, the equivalent quantity, $I_{\mathrm{BH}}^{0}$, is 


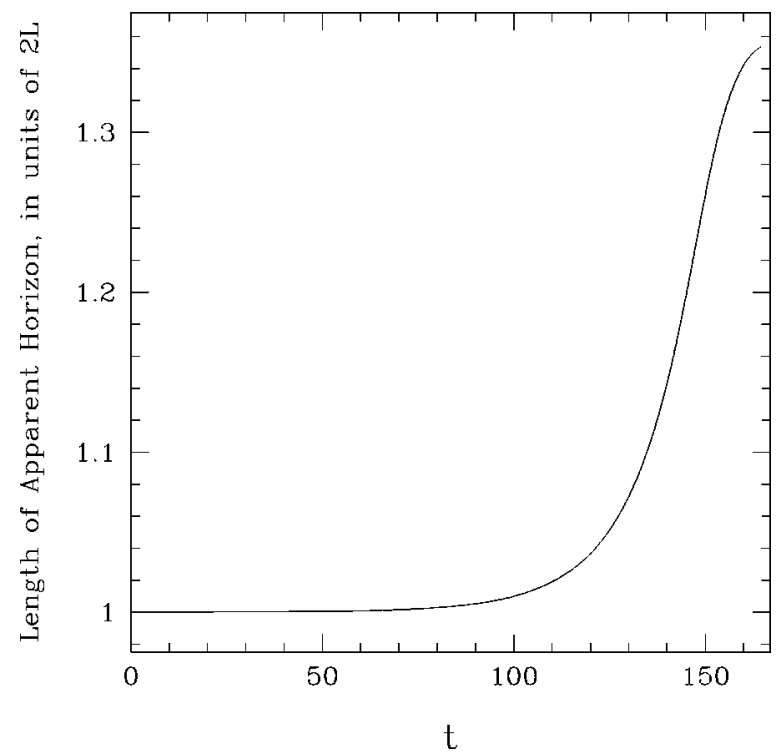

FIG. 5. The proper length of the apparent horizon curve in the $(r, z)$ plane (between $z=0$ and $z=L)$ as a function of time, from the $h / 4$ evolution of a perturbed black string with $L=1.4 L_{c}$.

$$
I_{\mathrm{BH}}^{0}=\frac{72}{R_{\mathrm{AH}}^{4}},
$$

where, in both of the above expressions, $R_{\mathrm{AH}}$ is the areal radius of the horizon. In Fig. 6 we plot the normalized quantity

$$
I^{0 N} \equiv \frac{I}{I_{\mathrm{BS}}^{0}}=\frac{I R_{\mathrm{AH}}^{4}}{12}
$$

evaluated on the apparent horizon of our numerical solution of the unstable spacetime- $I^{0 N}$ is 1 for a black string, and 6 for a black hole. The figure shows that, as judged by $I^{0 N}$, the part of the apparent horizon that is forming a long neck always resembles a black string - the part that is forming a bulge, however, has a value of $I^{0 N}$ tending towards that corresponding to a black hole. At $R_{\max }, I^{0 N}$ has only reached $\sim 5$ by the time the simulation ends; however, the behavior of $R_{\max }$ seen in Fig. 2 suggests that the growth in the normalized curvature invariant, though slowing down, should continue. Figure 6 also demonstrates the grid-stretching problems that we surmise are causing the code to eventually crash-in that plot we use the coordinate $z$ as the horizontal axis, and observe that the relatively small region where $I^{0 N}$ $\approx 1$ corresponds to the long neck in Fig. 4. In particular, in the vicinity of the "neck," $g_{z z}$ becomes quite large, as do its derivatives.

Finally, in Fig. 7 we show plots of the approximate event horizon (as described in Sec. II C 1), together with the apparent horizon for the simulation. The results shown in the plot suggest that our computed apparent horizon is an excellent approximation to the event horizon, at least at early times (not much can be said regarding the late time behavior of the event horizon, as the spacetime has not settled down to a stationary state when the simulation ends).

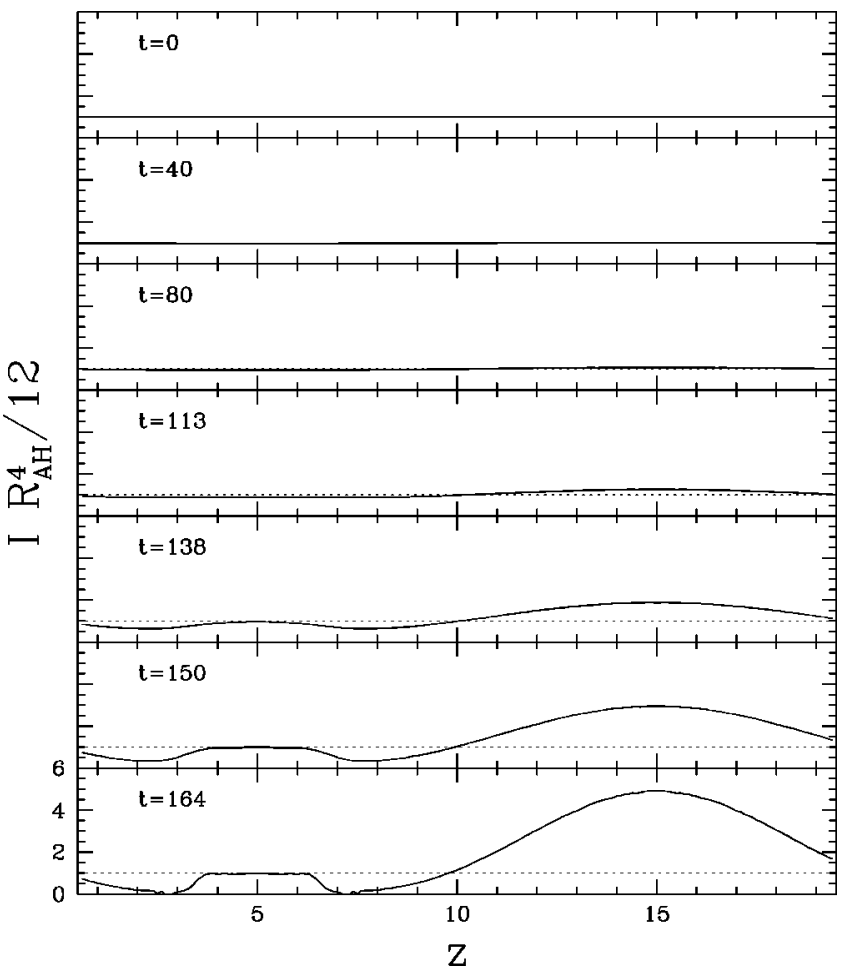

FIG. 6. The normalized Kretschmann invariant $I^{0 N} \equiv I R_{\mathrm{AH}}^{4} / 12$ (14), evaluated on the apparent horizon of the perturbed black string spacetime with $L=1.4 L_{c}(h / 4)$, at the same times as shown in the embedding diagram plots (Fig. 4). Note however, that here the horizontal axis is the coordinate $z$, and in particular the flat region of the curve between $z \approx 3.5$ and $z \approx 6.5$ in the last frame corresponds to the long, thin neck region shown in the embedding diagram plot. This demonstrates the rather severe "grid-stretching" problems we have then. For the static black string spacetime, $I^{0 N}=1$ (shown for reference as a dotted line in the figure), while for a static 5D spherical black hole it evaluates to 6 . This diagram therefore also supports the conclusion that at late (simulation) times the solution is tending towards a configuration describable as a sequence of black holes connected by thin black strings.

\section{CONCLUSIONS}

We have performed a preliminary numerical study of the instability of the 5-dimensional black string. Coordinate pathologies prevent us from definitively identifying the final end-state(s) of an unstable black string. This claim is supported by the fact that the code crashes at very nearly the same time at varying resolution, and that curvature invariants remain well behaved throughout the evolution. The former suggests that a numerical instability is not responsible for the crash, while the latter indicates that a physical singularity is probably also not to blame. Despite the premature termination of the simulation, we find evidence that the spacetime evolves towards a configuration that looks like a sequence of black holes connected by thin black strings, and characterized by an expansion of the string direction. Since the spacetime is still fairly dynamical at the time our simulations end, we cannot deduce how close this state is to a final configuration. Nevertheless, the dynamical behavior observed is sufficiently robust for some comments to be made. For instance, 


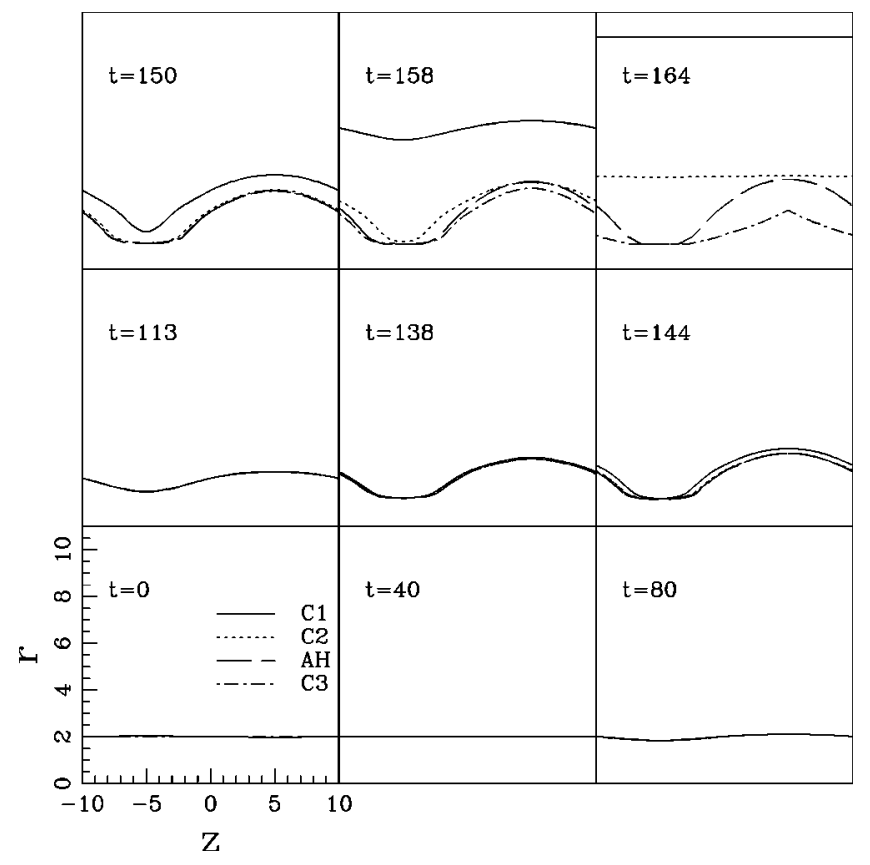

FIG. 7. Plots of the apparent horizon (labeled $\mathrm{AH}$ ) and estimates of the event horizon location $(\mathrm{C} 1, \mathrm{C} 2$ and $\mathrm{C} 3)$ in coordinate space (in contrast to the embedding coordinates used in Fig. 4), from the evolution of a perturbed black string with $L=1.4 L_{c}$, computed at resolution $h / 4$. Here, the $\mathrm{C} 1(\mathrm{C} 2)$ curve marks the inward-directed past light cone of the surface $r=10(r=4)$ at $t=164$. C3 denotes the outward-directed past of a surface just inside the apparent horizon at $t=164$. Thus, moving backwards in time, these curves should asymptote towards the event horizon of the spacetime. These plots suggest that for most of the evolution (at least), the apparent horizon is an excellent approximation to the event horizon.

the results are not inconsistent with Gregory and Laflamme's conjecture that the solution bifurcates into a sequence of black holes-indeed, we can suggest at least two mechanisms by which this could occur:

(1) Via a thinning neck that eventually vanishes, if the trend seen in the simulation continues. Note that this would require (a) that the proper length of the string continues to grow, in order not to violate area theorems, and (b) that the thin string be non-uniform, for otherwise it would be subject to a further Gregory-Laflamme-like instability.

(2) Via a sequence of Gregory-Laflamme instabilities, if the thin neck stays "close" to a uniform black string, since the neck's length is beyond the critical one for a string with an effective mass computed from the radius of the apparent horizon. In this case, one could envision a "cascade" of instabilities leading to the bifurcation.

We note that either scenario would not necessarily be inconsistent with Horowitz and Maeda's results, should the vanishing of the neck take infinite affine time as measured by local null generators of the horizon. At the same time, a continuation of the observed trend would argue against achieving a stationary solution with a mild dependence on the string dimension (i.e. a small value of $\lambda$ ), as found in perturbative calculations [10]. For then, the rather extreme thinning/bulging that we see must be transient behavior that is "further" from the end-state than the perturbed black string was.

A more complete exposition of the nature of unstable black string evolution would appear to require coordinate conditions able to adapt to solution features that develop at late times-that is, in a manner that does not introduce severe metric gradients that are not correlated with large gradients in physical quantities. For example, it may help to replace the fixed-lapse slicing with maximal slicing, which enforces that the divergence of the local, spatial volume element be zero. Another, perhaps even more crucial option, would be to introduce a $z$ component to the shift vector that keeps $g_{z z}$ close to (or exactly) unity throughout the evolution. These options are currently under investigation.

Additionally, it would be interesting to explore a wider range of initial conditions describing "perturbed" black strings than that considered here. For example, the imposed $z$ periodicity implies that the equivalent, uncompactified space time consists of identical spherical-black-hole/black-string segments at late (intermediate) times. It would be instructive to see what happens should we break this symmetry, by making $L \gg L_{c}$, and then introducing some higher-wavelength perturbation similar to $q>1$ in Eq. (13), but with more asymmetry in the initial data (note that this would be more computationally demanding). Finally, it would be very interesting to study the evolution of the solutions recently found by Wiseman [11] and mentioned in the Introduction. These configurations actually correspond to stationary solutions, and their perturbative stability, or otherwise, is currently not known. Since Wiseman shows that his solutions cannot be the end-states conjectured by Horowitz and Maeda, it is important to understand their behavior, since if they are stable they may well represent physically meaningful states, while if unstable, they may be difficult to attain via dynamical evolution. An interesting observation from our simulations, to the length so far achieved, is that they do not display a conical "waist" like those presented in [11] and further analyzed in [27].

\section{ACKNOWLEDGMENTS}

We gratefully acknowledge support from the following agencies, institutes and grants: NSERC, NSF PHY-0099568, The Canadian Institute for Advanced Research, The Canadian Institute for Theoretical Astrophysics, The Pacific Institute for Mathematical Sciences, The Government of the Basque Country, The Izaak Walton Killam Fund and Caltech's Richard Chase Tolman Fund. Computations were performed on (i) the vn.physics.ubc.ca cluster which was funded by the Canadian Foundation for Innovation (CFI) and the BC Knowledge Development Fund; (ii) LosLobos at Albuquerque High Performance Computing Center; (iii) the high-performance computing facilities within LSU's Center for Applied Information Technology and Learning, which is funded through Louisiana legislative appropriations, and (iv) the MACI cluster at the University of Calgary, which is funded by the Universities of Alberta, Calgary, Lethbridge and Manitoba, and by C3.ca, the Netera Alliance, CANA- 
RIE, the Alberta Science and Research Authority, and the CFI. We would like to thank G. Horowitz, W.G. Unruh, R. Wald, R. Myers, T. Wiseman and B. Kol for stimulating discussions.

\section{APPENDIX A: INITIAL DATA SOLVER}

We solve the set of coupled constraint equations (2),(3) via an iterative procedure, where at each substep of the iteration we solve a single equation for one of $g_{r r}, k_{\theta \theta}$ or $k_{r r}$, assuming that the values of the other variables are known. We iterate this process until the residuals of all the equations are simultaneously below a certain tolerance-a typical value is $10^{-5}$. The overall iteration is initialized using values corresponding to an unperturbed black string solution. We now provide a few more details concerning the solution of each of the constraint equations.

The equations are discretized on a uniform grid of points $\left(x_{i}, z_{j}\right)$ with $i=1, \ldots, N_{x}$, and $j=1, \ldots, N_{z}$ [recall that, from Eq. (7), the radial coordinate, $r$, is related to $x$ by $r$ $=x /(1-x)]$. We first consider the Hamiltonian constraint (2) which, in the coordinate system we have adopted, has the following form:

$$
\begin{aligned}
& F_{1} \frac{\partial g_{r r}}{\partial x}+F_{2} g_{r r} \frac{\partial^{2} g_{r r}}{\partial z^{2}}+F_{3} g_{r r} \frac{\partial g_{r r}}{\partial z}+F_{4}\left(\frac{\partial g_{r r}}{\partial z}\right)^{2}+F_{5}\left(g_{r r}\right)^{2} \\
& \quad+F_{6} g_{r r}=0 .
\end{aligned}
$$

Here, the $F_{m}, m=1, \ldots, 6$, are functions that generally depend on all the metric coefficient and their derivatives except $g_{r r}$ (and its derivatives). We discretize this equation to second order in the mesh spacing using a difference approximation centered at the points $\left(x_{i+1 / 2}, z_{j}\right)$. Because of the discretization used and the form of Eq. (A1), we can solve the resulting set of equations "line-by-line" in $x$, starting at the inner boundary, $i=1$, which is chosen well within the horizon of the string (typically at $r=M$ ), and where the boundary values, $\left[g_{r r}\right]_{1, j}, j=1, \ldots, N_{z}$, are those corresponding to an unperturbed black string. As we integrate outwards in $x$, the determination of the $i$ th line of unknowns, $\left[g_{r r}\right]_{i, j}, j$ $=1, \ldots, N_{z}$, involves the solution of an $N_{z}$-dimensional, non-linear, cyclic (because of the $z$ periodicity), tridiagonal system that we solve using Newton's method and a cyclic tridiagonal linear solver [28].

We now direct attention to the $r$ momentum constraint, which, viewed as an equation for $k_{\theta \theta}$, has the form

$$
G_{1} \frac{\partial k_{\theta \theta}}{\partial x}+G_{2} k_{\theta \theta}+G_{3}=0
$$

where the $G_{m}, m=1,2,3$ do not depend on $k_{\theta \theta}$ or its derivatives. We note that there is complete decoupling in the $z$ direction in this case; in effect, we have to solve an ODE along each $z=$ const line. We again discretize using secondorder finite difference techniques, fix the boundary values $\left[k_{\theta \theta}\right]_{N_{x}, j}, j=1, \ldots, N_{z}$, at $x=1\left(i_{o}\right)$ using the unperturbed black string solution, then solve for the remaining unknowns, marching inwards in $x$.
Finally, the $z$ component of the momentum constraint, which fixes $k_{r r}$, has the form

$$
H_{1} \frac{\partial k_{r r}}{\partial z}+H_{2} k_{r r}+H_{3}=0,
$$

where the $H_{m}, m=1,2,3$ are independent of $k_{r r}$ and its derivatives. This equation is solved analogously to the $r$ momentum constraint, but now using a discretization that is centered at points $\left(x_{i}, z_{j+1 / 2}\right)$. "Boundary values," $\left[k_{r r}\right]_{i, 1}, i=1, \ldots, N_{x}$, are specified along the line $z=z_{\min }$, again using corresponding values from the black string solution, and the integration proceeds for $j=2,3, \ldots, N_{z}$.

\section{APPENDIX B: FINDING APPARENT HORIZONS}

We use a flow, or level-set method to search for apparent horizons within $t=$ const spatial slices of the spacetime. We restrict our search to simply connected apparent horizons that are periodic in z. Such an apparent horizon can be described by a curve in the $(r, z)$ plane, which we define to be the level surface $F=0$ of the function

$$
F(r, z)=r-R(z) .
$$

In other words, the apparent horizon will be given by the curve $r=R(z)$. The apparent horizon is the outermost, marginally trapped surface; hence, we want to find an equation for $R(z)$ such that the outward null expansion, normal to the corresponding surface $F=0$, is zero. To this end, we first construct the unit spatial vector $s^{a}$, normal to $F=$ const:

$$
s^{a}=\frac{g^{a b} F_{, b}}{\sqrt{g^{c d} F_{, c} F_{, d}}} .
$$

Then, using $s^{a}$ and the $t=$ const hypersurface normal vector $n^{a}$, we can construct future-pointing outgoing $(+)$ and ingoing $(-)$ null vectors

$$
\ell^{a} \pm=n^{a} \pm s^{a} .
$$

The normalization of the null vectors is (arbitrarily) $\ell_{+}^{a} \ell_{-a}$ $=-2$. The outward null expansion $\theta_{+}$is then the divergence of $\ell_{+}^{a}$ projected onto an $F=$ const surface:

$$
\theta_{+}=\left(g^{a b}-s^{a} s^{b}\right) \nabla_{b} \ell_{+a} .
$$

Substituting expressions (B1) and (B2) into Eq. (B4), with $\theta_{+}=0$, provides us with an ordinary differential equation for $R(z)$.

Initially we solved Eq. (B4) via a "shooting" methodgiven a guess for $R(0)$, and assuming that $R^{\prime}(0)=0$ (where a prime denotes differentiation with respect to $z$ ), we integrate the equation to $z=z_{L}$, and repeat the process until we find a solution where $R\left(z_{L}\right)=R(z)$ and $R^{\prime}\left(z_{L}\right)=0$. An efficient sequence of guesses can be generated using a bisection search, as the qualitative behavior of the solution is different depending upon whether the initial guess for $R(0)$ is inside or outside the apparent horizon. 
Since the shooting method is difficult to extend to a parallel implementation in an efficient way, we opted to use the following point-wise relaxation method (or flow method) to determine $R(z)$. We supply an initial guess, $R_{0}(z)$, for the entire function $R(z)$, and then iterate the following equation until the norm of the expansion $\theta_{+}(z)$ of $F(0)$ is below some desired threshold (in our runs we have typically set it to $10^{-3} h$, where $h$ is the basic scale of discretization):

$$
\Delta R_{n}(z) \equiv R_{n+1}(z)-R_{n}(z)=-\theta_{+}(z) \Delta \tau .
$$

Here, $R_{n}(z)$ is the solution after the $n^{\text {th }}$ iteration. Equation (B5) can be viewed as an explicit discretization of a parabolic evolution equation for $R(z, \tau)$, where $\tau$ is "time" and $\Delta \tau$ is the time step for the evolution (the parabolic nature of the equation is evident when $\theta_{+}$is expanded in terms of $R(z)$ via Eq. (B4) - for brevity we do not give the explicit form of the equation here). Thus, for stability $\Delta \tau$ must be chosen to be less than $\Delta z^{2}$.

Given a "reasonable" initial guess $R_{0}(z)$, one can see how iteration of Eq. (B5) will cause $R_{n}(z)$ to "flow" to the apparent horizon: if $R_{n}(z)$ is outside of the apparent horizon, then typically the expansion $\theta_{+}(z)$ will be positive there, causing $R_{n+1}(z)$ to decrease towards the apparent horizon, and vice versa if $R_{n}(z)$ is inside the apparent horizon. We use $R(z)=2$ as the initial guess at $t=0$; after $t=0$ we search for the apparent horizon every $N$ time steps (where $N$ is typically in the range 10-30), and use the shape found at the previous search as the initial guess for the next search. Usually, on the order of tens to thousands of iterations of Eq. (B5) are required to solve for $R(z)$ to within a level of accuracy such that the approximate solution is roughly within a mesh spacing of the exact solution (as estimated in a few specific calculations by solving Eq. (B5) close to machine precision). A single iteration of Eq. (B5) can be computed very rapidly relative to the time taken to compute a metricevolution step; however, in a parallel environment, if thousands of iterations are needed on a regular basis (which is so at late times during the evolution of an unstable black string), the apparent horizon finder becomes a slight speed bottleneck in the code, due to the time it takes to communicate the results of each iteration amongst the processors involved.

\section{APPENDIX C: FINDING (APPROXIMATE) EVENT HORIZONS}

Here we describe one method we use, following [25], to locate approximations to event horizons. This method involves locating the boundary of the causal past of some $r$ $=$ const surface of the spacetime by following radial null geodesics.

We write the geodesic equation in Lagrangian form:

$$
\mathcal{L}=g_{t t}\left(t^{\prime}\right)^{2}+2 g_{t r} t^{\prime} r^{\prime}+g_{r r}\left(r^{\prime}\right)^{2}+2 g_{r z} r^{\prime} z^{\prime}+g_{z z}\left(z^{\prime}\right)^{2},
$$

where $\lambda$ is the affine parameter along the geodesic, and a prime denotes differentiation with respect to $\lambda$. Since we are interested in null trajectories, we set $\mathcal{L}=0$. For radial, ingoing geodesics, we have $\theta^{\prime}=\phi^{\prime}=0$, and thus the geodesic equations reduce to the set:

$$
\begin{gathered}
\dot{r}=\frac{\alpha}{\sqrt{g_{r r}}}-\beta, \\
\dot{\lambda}=\frac{2 \alpha \sqrt{g_{r r}}}{\Pi_{r}},
\end{gathered}
$$

where the dot denotes a derivative with respect to coordinate time, and $\Pi_{r}=\partial \mathcal{L} / \partial r^{\prime}$. Then, starting at a certain value of $r=r_{0}$, and for each grid point along the $z$ direction, Eqs. (C2) are integrated backwards in time using a second order Runge-Kutta scheme.

Following null geodesics along $z=$ const lines does not guarantee that we are tracing the causal past of $r=r_{0}$, though for the spacetimes studied here, and the coordinate system used, this should offer a good approximation. Furthermore, although radial geodesics might not be the best choice, since the event horizon is an attractor, they will trace it accurately. (For related discussions of approximate event horizon location in the axisymmetric four-dimensional case, see [2931].)
[1] S. Chandrasekhar, The Mathematical Theory of Black Holes (Oxford University Press, Oxford, 1983).

[2] R. Gregory and R. Laflamme, Phys. Rev. Lett. 70, 2837 (1993).

[3] R. Gregory and R. Laflamme, Nucl. Phys. B428, 399 (1994).

[4] S. Hawking and G. Ellis, The Large Scale Structure of Spacetime (Cambridge University Press, Cambridge, England, 1973).

[5] G.T. Horowitz, J.M. Maldacena, and A. Strominger, Phys. Lett. B 383, 151 (1996).

[6] N. Itzhaki, J.M. Maldacena, J. Sonnenschein, and S. Yankielowicz, Phys. Rev. D 58, 046004 (1998).

[7] O. Aharony, S.S. Gubser, J.M. Maldacena, H. Ooguri, and Y. Oz, Phys. Rep. 323, 183 (2000).
[8] G.T. Horowitz and V.E. Hubeny, Phys. Rev. D 62, 024027 (2000).

[9] G. Horowitz and K. Maeda, Phys. Rev. Lett. 87, 131301 (2001).

[10] S. Gubser, Class. Quantum Grav. 19, 4825 (2002).

[11] T. Wiseman, Class. Quantum Grav. 20, 1177 (2003).

[12] B. Kol, hep-th/0208056.

[13] W. Unruh and R. Wald (in preparation).

[14] J. Geddes, Phys. Rev. D 65, 104015 (2002).

[15] C.W. Misner, K.S. Thorne, and J.A. Wheeler, Gravitation (Freeman, New York, 1973).

[16] J.W. York, Jr., in Sources of Gravitational Radiation, edited by L. Smarr (Cambridge University Press, Seattle, 1979).

[17] L. Lehner, Class. Quantum Grav. 18, R25 (2001). 
[18] H.A. Shinkai and G. Yoneda, Class. Quantum Grav. 19, 1027 (2002).

[19] B. Kelly et al., Phys. Rev. D 64, 084013 (2001).

[20] J. Thornburg, Class. Quantum Grav. 4, 1119 (1987).

[21] E. Seidel and W.M. Suen, Phys. Rev. Lett. 69, 1845 (1992).

[22] L. Lehner, M. Huq, and D. Garrison, Phys. Rev. D 62, 084016 (2000).

[23] The CACTUS Computational Toolkit, http:// www.cactuscode.org

[24] H.O. Kreiss and J. Oliger, Method for the Approximate Solution of Time Dependent Problems (GARP Publication Series,
Geneva, 1973).

[25] J. Libson et al., Phys. Rev. D 53, 4335 (1996).

[26] R. Wald and S. Iyer, Phys. Rev. D 44, 3719 (1991).

[27] B. Kol and T. Wiseman, hep-th/0304070.

[28] W.H. Press, S.A. Teukolsky, W.T. Vetterling, and B.P. Flannery, Numerical Recipes (Cambridge University Press, Cambridge, England, 1992).

[29] S. Hughes et al., Phys. Rev. D 49, 4004 (1994).

[30] J. Masso et al., Phys. Rev. D 59, 064015 (1999).

[31] S.A. Caveny and R.A. Matzner, gr-qc/0303099. 